\title{
Creation of Novel Cell-Penetrating Peptides for Intracellular Drug Delivery Using Systematic Phage Display Technology Originated from Tat Transduction Domain
}

\author{
Haruhiko Kamada, ${ }^{*, a}$ Takayuki Oкамото, ${ }^{b, c}$ Maki Kawamura, ${ }^{a, b}$ Hiroko Shibata, ${ }^{a, b}$ Yasuhiro Abe, ${ }^{a, b}$ \\ Akiko Ohkawa, ${ }^{a, b}$ Tetsuya Nomura, ${ }^{a, b}$ Masaki Sato, ${ }^{a, b}$ Yohei Mukai, ${ }^{a, b}$ Toshiki Sugita,${ }^{a, b}$ \\ Sunao ImaI,${ }^{a, b}$ Kazuya Nagano, ${ }^{a, b}$ Yasuo Tsutsumi,${ }^{a, b}$ Shinsaku Nakagawa, ${ }^{b}$ \\ Tadanori MAYumi, ${ }^{d}$ and Shin-ichi TsunODA ${ }^{a}$ \\ ${ }^{a}$ Laboratory of Pharmaceutical Proteomics, National Institute of Biomedical Innovation; 7-6-8 Asagi, Saito, Ibaraki, \\ Osaka 567-0085, Japan: ${ }^{b}$ Department of Biopharmaceutics, Graduate School of Pharmaceutical Sciences, Osaka \\ University; 1-6 Yamadaoka, Suita, Osaka 565-0871, Japan: ${ }^{c}$ Department of Molecular Pathobiology, Mie University \\ School of Medicine; 2-174 Edobashi, Tsu, Mie 514-8507, Japan: and ${ }^{d}$ Department of Cell Therapeutics, Graduate School \\ of Pharmaceutical Sciences, Kobe Gakuin University; 518 Arise, Ikawadani, Nishi-ku, Kobe 651-2180, Japan. \\ Received March 14, 2006; accepted November 13, 2006; published online November 16, 2006
}

\begin{abstract}
Many biologically active proteins need to be delivered intracellularly to exert their therapeutic action inside the cytoplasm. Cell penetrating peptides (CPPs) have been developed to efficiently deliver a wide variety of cargo in a fully biological active form into a range of cell types for the treatment of multiple preclinical disease models. To further develop this methodology, we established a systematic approach to identify novel CPPs using phage display technology. Firstly, we screened a phage peptide library for peptides that bound to the cell membrane. Secondly, to assess functionality as intracellular carriers, we recombined cDNAs of binding peptides with protein synthesis inhibitory factor (PSIF) to create fusion proteins. Randomly chosen clones were cultured and expression of peptide-PSIF fusion proteins induced, followed by screening of protein synthesis activity in cells. Using this systematic approach, novel and effective CPPs were rapidly identified. We suggest that these novel cell-penetrating peptides can utilized as drug delivery tools for protein therapy or an analytical tool to study mechanisms of protein transduction into the cytoplasm.
\end{abstract}

Key words cell penetrating peptide; phage display; Tat

Many biologically active compounds, including a variety of large molecules, need to be delivered intracellularly to exert their therapeutic action inside the cytoplasm or within the nucleus or other specific organelles. An important requirement in the use of proteins in this context (ex. kinases, phosphatases, transcriptional factors) is the ability of these molecules to efficiently penetrate across the cell membrane. However, the plasma membrane of cells is largely impermeable to proteins and peptides. Recently, it was discovered that certain short peptide sequences, composed mostly of basic, positively charged amino acids (e.g. Arg, Lys and His), have the ability not only to transport themselves across cell membranes, ${ }^{1-3)}$ but also to carry attached molecules (proteins, DNA, or even large metallic beads) into cells. $\left.{ }^{4}{ }^{6}\right)$ These basic sequences are now commonly known as protein transduction domains or cell-penetrating peptides (CPPs) and have been successfully employed to transport cargo proteins across a variety of cell membranes. ${ }^{7)}$ Cellular delivery using CPPs has several advantages over conventional techniques; indeed, it is efficient across a range of cell types and can be applied to cells en masse. ${ }^{8)}$

It has been proposed that the Tat transduction domain of HIV is first endocytosed into a caveola compartment and secondarily released into the cytoplasm, following vesicle disruption. ${ }^{9)}$ Once CPP binds to the cell surface heparan sulfate proteoglycan (HSPG), the CPP-fused protein is internalized via a lipid raft-mediated pathway. Additionally, the mechanisms responsible for CPP mediated cargo internalization estimated with regard to enter the cells via macropinocytosis ${ }^{10)}$ and/or through clathrin-mediated endocytosis, ${ }^{11)}$ or possibly via an unknown alternative mechanism. In spite of some common features of these peptides, particularly their highly cationic nature, their structural diversity has fuelled the idea that the penetrating mechanism is not the same for CPPs of different types. As such, the mechanism(s) of internalization of CPPs has not been resolved yet. ${ }^{7)}$

Given the potency of the Tat-derived CPPs in mediating the cellular uptake of small and large macromolecular cargos, as demonstrated within the last few years, a large number of laboratories have exploited this system as a tool for transcellular penetration of cultured cells. ${ }^{12)}$ Most of these applications are based on the fusion of the protein transduction domain of Tat to the protein of interest, either at the Nor C-terminus, followed by addition of the recombinant fusion protein to the culture medium of the cells of interest. It is clear that CPPs are novel vehicles for the rapid translocation of cargo into cells, and exhibit the properties that make them potential drug delivery agents. ${ }^{13)}$ However, there are problems in respect to a decrease in the rate and efficiency of translocation for large proteins that has not yet been overcome. Accordingly, a large number of different CPPs have been explored to promote translocation of various types of useful cargo, ranging from small molecules to proteins and large supramolecular particles, with great efficiency and reasonable velocity.

We previously showed that the gene III proteins (pIII) of M13 filamentous phage could be used to display mutant protein, with these modified proteins showing fully functional binding to receptor and consequent biological activities. ${ }^{14,15)}$ Recently, we established a novel whole cell panning method, 
which selected cell adhesive phage-displayed peptides and, subsequently, a cohort of these peptides having cell penetrating qualities via the use of PSIF (protein synthesis inhibitory factor). In this study, we constructed a Tat-based mutant peptide library using this phage display system. Moreover, we demonstrated the direct selection of a unique cell-binding activity utilizing whole cell panning methods and the screening of internalizing peptide using peptide-PSIF fusion protein.

\section{MATERIALS AND METHODS}

Cell Line Human epidermoid carcinoma A431 cells were grown in Dulbecco's Modified Eagle's Medium (DMEM) supplemented with $10 \%$ fetal calf serum (FCS) in $5.0 \% \mathrm{CO}_{2}$ at $37^{\circ} \mathrm{C}$. Human adenocarcinoma Hela cells were grown in DMEM supplemented with NEAA and 10\% FCS. Chinese hamster ovary (CHO)-K1 cells were grown in Ham's F12K medium supplemented with $2 \mathrm{~mm}$ L-glutamine and $10 \%$ FCS.

Preparation of Phage Peptide Library Primers shown below and used in library construction were purchased from Hokkaido System Science Inc. The phage-display vector pCANTAB-5E was used as a phagemid vector for the generation of the peptide-pIII fusion gene repertoires (Fig. 1). To construct a DNA fragment library encoding 13 amino acid peptides, primers P-oligo1 and P-ologo2 were annealed and elongated with the Klenow fragment in the presence of nucleotide triphosphates. These cDNA-encoding peptide library products were purified with QIAquick ${ }^{\circledR}$ Gel Extraction Kit (QIAGEN) and used as templates for PCR with primers pCANTAB-Hind and Not I Ext to generate the pIII fusion gene repertoires. The peptide-encoding genes were digested with the restriction enzymes HindIII and NotI, agarose gelpurified, and ligated into pCANTAB-5E, which was cut with the same restriction enzyme. The ligated products were electroporated into E. coli TG1 cells, plated on modified LB medium (Invitrogen) containing $2 \%$ glucose and $50 \mu \mathrm{g} / \mathrm{ml}$ ampicillin, and then incubated overnight at $37^{\circ} \mathrm{C}$. The clones were scraped off the plates into $2 \mathrm{YT}$ medium with $10 \%$ glyc- erol and subsequently stored at $-70^{\circ} \mathrm{C}$. P-oligo $1 ; 5^{\prime}$-GAT TAC GCC AAG CTT TGG AGC CTT TTT TTT GGA GAT TTT CAA CGT GAA AAA ATT ATT ATT CGC AAT TCC TTT AGT TGT TCC TTT CTA TGC GGC CCA GCC GGC CAT GGC C-3', P-oligo2; 5'-CGG CGC ACC TGC GGC CGC SNN SNN CGG SNN SNN SNN CTG SNN SNN SNN SNN SNN ACC GGC CAT GGC CGG CTG GGC CGC ATA GAA AGG-3', pCANTAB-Hind; 5'-GGA AAC AGC TAT GAC CAT GAT TAC GCC AAG-3', Not I Ext; 5' GCG GCC TTG TCA TCG TCA TCC TTG TAG TCT GCG GCC GC-3'.

Rescue of Peptide-Phage To rescue the peptide-phage library, 11 of $2 \mathrm{YT}$ medium, containing $2 \%$ glucose, and $100 \mu \mathrm{g} / \mathrm{ml}$ of ampicillin, was inoculated from the glycerol stock library. The culture was shaken at $37^{\circ} \mathrm{C}$ until OD $600 \mathrm{~nm}=0.4$ and $3.2 \times 10^{8}$ plaque forming units of M13KO7 helper phage (Invitrogen) were added. After $30 \mathrm{~min}$ incubation at room temperature with shaking, the culture was centrifuged and the pellet recovered. The pellet was then incubated with $50 \mu \mathrm{g} / \mathrm{ml}$ of kanamycin and $100 \mu \mathrm{g} / \mathrm{ml}$ of ampicillin within 2YT medium and grown for $6 \mathrm{~h}$ at $37^{\circ} \mathrm{C}$. The phage was purified by standard polyethylene glycol precipitation and filtration with a $0.45 \mu \mathrm{m}$ PVDF filter (Millipore). Peptide-phage which did not express the objective peptide were removed by a FLAG panning method, as described previously.

Biopanning Method We used a slightly modified procedure from that found in the literature. Briefly, $1.0 \times 10^{6} \mathrm{~A} 431$ cells were harvested in 6 well culture plates and incubated for $24 \mathrm{~h}$ at $37^{\circ} \mathrm{C}$ within a $5.0 \% \mathrm{CO}_{2}$ incubator until the logarithmic phase of growth was reached. The culture plates were washed with PBS 3 times and 2\% BSA Opti-MEM ${ }^{\circledR}$ (Invitrogen) added $2 \mathrm{~h}$ prior to the addition of the peptide-phage. Cells were incubated with the peptide-phage library for $2 \mathrm{~h}$ at $37^{\circ} \mathrm{C}$ with shaking every $15 \mathrm{~min}$ during the round of panning. Following this, the cells were washed twenty times with PBS at room temperature. After washing, the cells were lysed with $1 \mathrm{ml}$ of $100 \mathrm{~mm} \mathrm{HCl}$ and neutralized with $0.5 \mathrm{ml}$ of $1 \mathrm{M}$ Tris- $\mathrm{HCl}, \mathrm{pH}$ 8.0. One-hundred microliters lysate was used

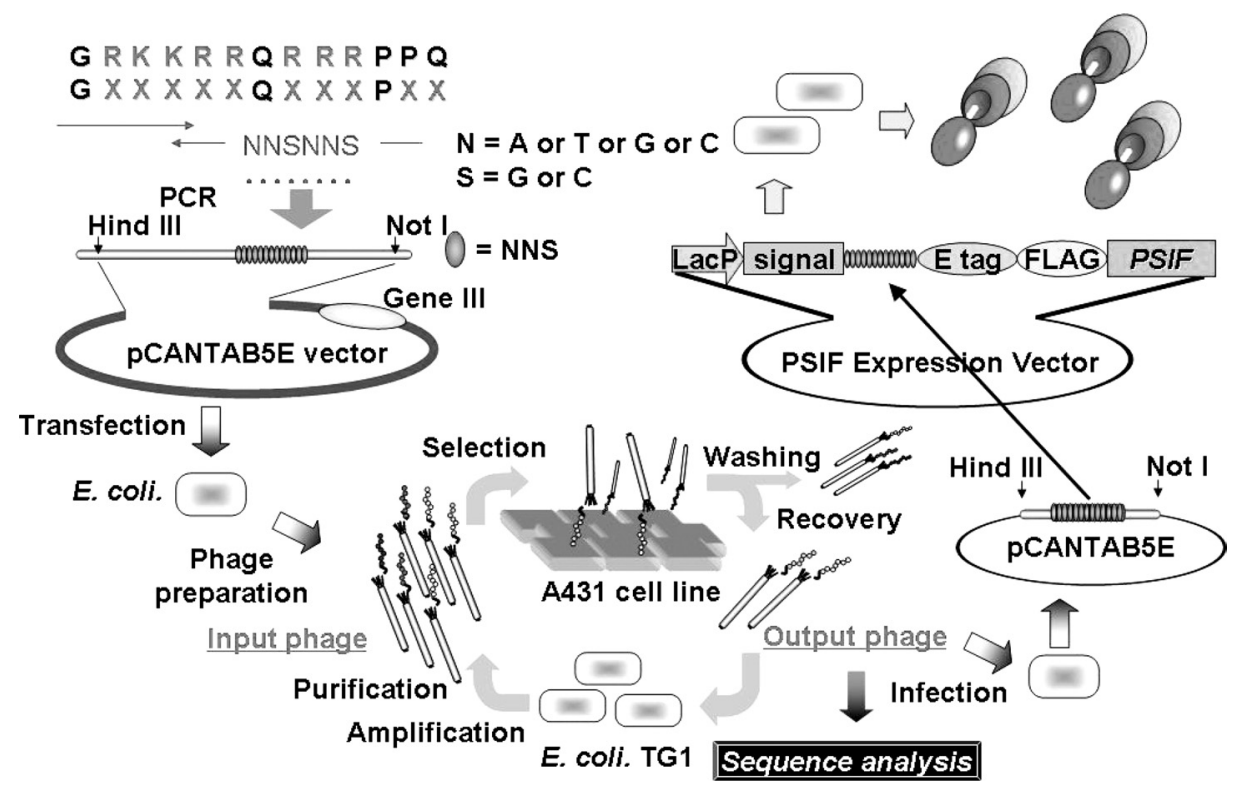

Fig. 1. Schema for Screening CPPs 
to infect $0.3 \mathrm{ml}$ of $E$. coli TG1 cells, with phage being rescued as described above and used in the next round of selection.

Expression and Purification of a Peptide-PSIF Fusion Protein Protein synthesis inhibitory factor (PSIF, PE fragment) is an approximately $40 \mathrm{kD}$ fragment of the bacterial exotoxin (GenBank Accession No. K01397) derived from Pseudomonas aeruginosa (ATCC strain No. 29260). PSIF lacks its cell binding domain, and is the truncated form of Pseudomonas aeruginosa exotoxin, which is a non-toxic protein outside of the cell. One (Dr. Tsunoda) of us cloned the cDNA for PSIF from Pseudomonas aeruginosa, Migula by PCR using the primer set 5'-GAT GAT CGA TCg cgg ccg caG GTG CGC CGG TGC CGT ATC CGG ATC CGC TGG AAC CGC GTG CCG CAg act aca aag acg acg acg aca aaC CCG AGG GCG GCA GCC TGG CCG CGC TGA CC-3' and 5'-GAT CGA TCG ATC act agt CTA cag ttc gtc ttt CTT CAG GTC CTC GCG CGG CGG TTT GCC GGG-3'. The fusion protein, denoted peptide-PSIF, consisted of peptide at the N-terminus and a PSIF at the C-terminus. First, the peptide gene containing phagemid vectors were recovered with QIAprep ${ }^{\circledR}$ Miniprep Kit (QIAGEN) and digested with HindIII and NotI. The peptide gene fragments were then subcloned into PSIF Expression Vector, which is modified from pCANTAB-5E. The fusion proteins were expressed and collected within the supernatant from $E$. coli TG1 cells, with the supernatant being used for cell viability assays.

Cell Viability A431 cells $\left(2.0 \times 10^{4}\right)$ were incubated with $35 \mu \mathrm{l} \mathrm{Opti-MM^{ \circledR }}{ }^{\circledR}$ and $10 \mu \mathrm{l}$ cycloheximide $(100 \mu \mathrm{g} / \mathrm{ml})$ in 96 well plates. Cells were treated with $5 \mu$ peptide-containing supernatant for $24 \mathrm{~h}$ and the cell viability was monitored by MTT assay. Twenty-four hours after addition of the peptides, $10 \mu \mathrm{l}$ of $5 \mathrm{mg} / \mathrm{ml}$ MTT (Dojindo) were added to each well and the cells were further incubated at $37^{\circ} \mathrm{C}$ for $4 \mathrm{~h}$. Subsequently, the insoluble formazan crystals were solubilized in a solution of $20 \%$ SDS containing $0.01 \mathrm{~N} \mathrm{HCl}$. Absorbance measurements were taken at $\lambda=595 \mathrm{~nm}$ with background subtracted at $\lambda=655 \mathrm{~nm}$. Each sample point was performed in duplicate.

FACS Analysis The specific cell binding activities of peptides towards A431, Hela, CHO-K1 cells were measured by FACScan (Becton Dickinson). Cells were grown in tissue culture flasks to late logarithmic phase. Culture medium was renewed $2 \mathrm{~h}$ prior to the addition of the peptide-phage. FITClabeled peptides were purchased from Genenet Co., Ltd. and $1 \times 10^{5}$ cells were incubated with FITC-labeled peptide for $3 \mathrm{~h}$ at $37^{\circ} \mathrm{C}$. For the endocytosis inhibitor assays, FACS analysis was performed after pre-treating A431 cell monolayers at $37^{\circ} \mathrm{C}$ with $10 \mathrm{~mm}$ methyl- $\beta$-cyclodextrin $(\mathrm{M} \beta \mathrm{CD}$; caveola-mediated endocytosis inhibitor) or amiloride (macropinocytosis inhibitor) in serum-free Minimal Essential Medium (MEM) for $30 \mathrm{~min}$, followed by a 1-h co-incubation with FITC labeled-peptide. After three washes with PBS, $0.25 \%$ trypsin solution (Gibco BRL) was added and incubated for $15 \mathrm{~min}$ to digest non-specific binding peptides. After three additional washes, cells were resuspended in $\mathrm{PBS} / 4 \%$ paraformaldehyde and analyzed using FACScan.

\section{RESULTS}

Construction of Phage Peptide Library and Quality
Check The pCANTAB-5E phagemid library used here has previously been screened successfully for mutant protein which binds to receptors. ${ }^{14,15)}$ Additionally, we previously reported the identification and characterization of a series of cationic peptides, similar to the CPP derived from Tat, which are able to penetrate large protein complexes into a wide variety of cells, including fibroblasts. Here, we made a novel phage peptide library, which altered ten amino acids within the Tat transduction domain (13 amino acids). The library of the TAT-based CPPs was made via the annealing and elongation of two mutated primers, followed by PCR amplification and cloning into a phage expression system The peptide-encoding cDNA library was placed into a phagemid vector and expressed as a fusion protein with phage coat protein, pIII. We confirmed the identity and sequence distribution of this phage peptide library by DNA sequencing (Table 1). In this context, eight clones which were sequenced showed independent sequences, highlighting the distribution of this phage peptide library.

Concentration of Binding Peptides with a Cell Panning Method The constructed peptide phage library was selected in vitro against A431 cells. Selection was performed as described in the Materials and Methods section, with a view to enriching for peptides displaying cell binding activity. With respect to the phage panning and amplification processes, which were repeated for one to four rounds, the output/input ratio was found to increase in a manner dependent on cycle number (Fig. 2). These results indicated that the peptides having an affinity for A431 cells were enriched gradually by this cell panning approach.

Identification of A431 Cell Binding Peptides Peptide clones that became internalized in A431 cells were isolated by four rounds of selection. In order to select only internalized phage-derived peptides, cells were incubated with super-

Table 1. Random TAT Peptide Library Sequence before Panning

\begin{tabular}{cc}
\hline \hline Clone & Sequence \\
\hline 1 & GMHINGQSNPPHA \\
2 & GGMHESQSHMPGD \\
3 & GTQAFLQQFEPWI \\
4 & GIKHSPQQISPRW \\
5 & GILCIQQDHQPLG \\
6 & GFKLSSQAVAPLQ \\
7 & GSIRAPQGDSPWP \\
8 & GTRHGIQTQPPNN
\end{tabular}

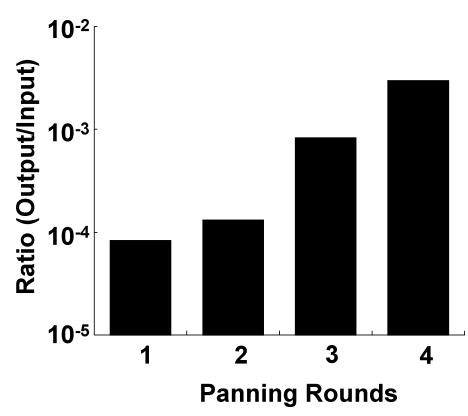

Fig. 2. Enrichment of Phage Clones by Biopanning

A431 cells were incubated with $4 \times 10^{10}$ titer phage. After washing with PBS, binding phages were recovered and the titer was determined. The index of enrichment was evaluated with input/output ratio. 


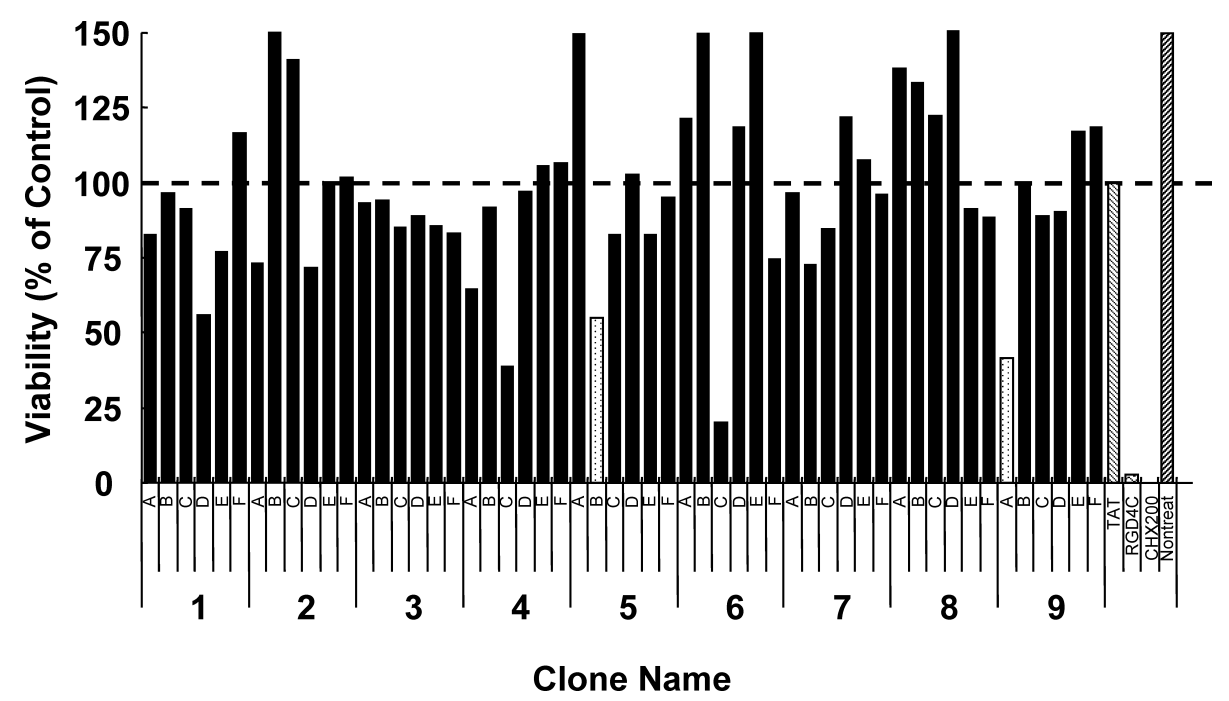

Fig. 3. Measurement of Penetrating Activity as an Index of Cytotoxicity to A431 Cells

PSIF-CPP fusion genes were transformed into TG1 cells, with resulting fusion proteins then recovered from the supernatant. The cytotoxicity of the supernatant was then assessed to examine the activity of the CPPs. Cell viability in response to fusion proteins was compared with that exhibited by exposure to the parent Tat13 peptide (cell viability $=100 \%$ ), and by exposure to $200 \mu \mathrm{g} / \mathrm{ml}$ cycloheximide (CHX; cell viability $=0 \%$ ).

natant collected from $E$. coli cells. After four rounds of selection, an enrichment in the order of $10^{5}$ was obtained. The random insert region of the single-stranded DNA from individual clones, following the fourth round of selection, was sequenced and the amino acid coding sequence deduced. To identify peptides capable of facilitating internalization, we developed a screening method using an M13 peptide phage display library. We incubated $1 \times 10^{6}$ A 431 cells, with $4 \times 10^{10}$ phage from a 13-mer peptide M13 phage display library for $2 \mathrm{~h}$ at $37^{\circ} \mathrm{C}$. Following four rounds of screening, we isolated phage from 28 plaques and determined the identity of the encoded peptides by DNA sequencing (Table 2). Twenty-eight of the peptide sequences were found in at least 15 independent plaques and were selected for further analysis.

Evaluation of Cell Penetrating Activity of CPPs Following creation of the peptide library (input phage library), we expressed these Tat-based CPPs as fusion proteins with PSIF. From this, 54 candidates were found that exhibited lower cytotoxic activity than the parent Tat peptide (data not shown). These results indicated that the penetrating activity is remarkably decreased as a consequence of random conversion of amino acids within the Tat transduction domain. In addition, we made the PSIF-fused peptide library after fourth round panning (Fig. 3). In screening this peptide library, a tryptophan-rich (GSSSWWQRWWPPW) peptide was identified (Table 2). However, this peptide did not exhibit cytotoxicity when recombined with PSIF. This result indicated that this tryptophan-rich peptide binds to the cell membrane but does not penetrate through to the cytoplasm. Next, we reconfirmed that fixation of the cells significantly affected the cellular distribution of peptides (Fig. 4). 435B peptide (GPFHFYQFLFPPV) and 439A peptide (GSPWGLQHHPPRT) showed internalization characteristics similar to those of the parent Tat peptide. These FITC-labeled peptides did not show cytotoxicity at a dose of $10 \mu \mathrm{M}$. However, the two another clone (434C and 436C peptide) does not TAT-derived peptide, which is not consist of 13 amino acid or occur the flameshift, respectively. So we were excluded these two clones from followed experiment.
Table 2. Random Tat Peptide Library Sequence after 4th Panning

\begin{tabular}{|c|c|}
\hline Clone & Sequence \\
\hline 1 & GPMESLQAFWPPW \\
\hline 2 & GSSSWWQRWWPPW \\
\hline 3 & GSSSWWQRWWPPW \\
\hline 4 & GVFLLKQVPQPSH \\
\hline 5 & GSSSWWQRWWPPW \\
\hline 6 & GRLWWLQLFEPGH \\
\hline 7 & GLRKVPQSVPPDM \\
\hline 8 & GSSSWWQRWWPPW \\
\hline 9 & GHFLKPQVLRPTR \\
\hline 10 & GQFMMRQYWPPVH \\
\hline 11 & GSSSWWQRWWPPW \\
\hline 12 & GSSSWWQRWWPPW \\
\hline 13 & GSSSWWQRWWPPW \\
\hline 14 & GLLKYQQWASPLC \\
\hline 15 & GYFWYDQPWQPEQ \\
\hline 16 & GRNHYIQRDNPVS \\
\hline 17 & GVFHVLQNAI PQY \\
\hline 18 & GSSSWWQRWWPPW \\
\hline 19 & GTMPNMQHHDPAR \\
\hline 20 & GSSSWWQRWWPPW \\
\hline 21 & GSSSWWQRWWPPW \\
\hline 22 & GSSSWWQRWWPPW \\
\hline 23 & GTRYLVQYLFPHL \\
\hline 24 & GRPATQQGLTPAR \\
\hline 25 & GYIGTYQQWNPPP \\
\hline 26 & GSSSWWQRWWPPW \\
\hline 27 & GSSSWWQRWWPPW \\
\hline 28 & GSSSWWQRWWPPW \\
\hline
\end{tabular}

Uptake of FITC-Labeled Peptides into Human and Murine Cells To address the question of whether 435B and 439A peptides were more active than the parent Tat peptide, peptides conjugated to FITC were constructed. Cellular uptake of both peptides were judged by flow cytometric analysis on human carcinoma A431 and Hela and CHO cells. Assuming that the surface-adsorbed 435B and 439A peptides were susceptible to tryptic degradation, we washed the cells five times with PBS and treated them with trypsin prior to assessing the amount of the internalized peptide. On A431 


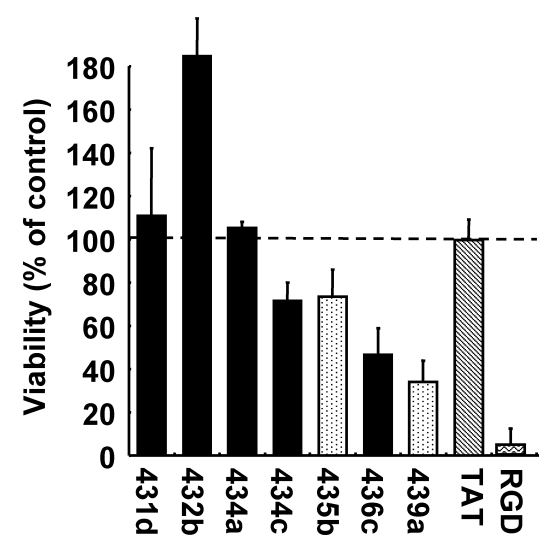

Fig. 4. Evaluation of Cell Penetrating Activity of Individual Clones

Cell penetrating activity was reconfirmed using the same method as that referred to in Fig. 3. Cell viability in response to fusion proteins was compared with that exhibited by exposure to the parent Tat 13 peptide (cell viability $=100 \%$ ), and by exposure to $200 \mu \mathrm{g} / \mathrm{ml}$ cycloheximide (CHX; cell viability $=0 \%$ ).

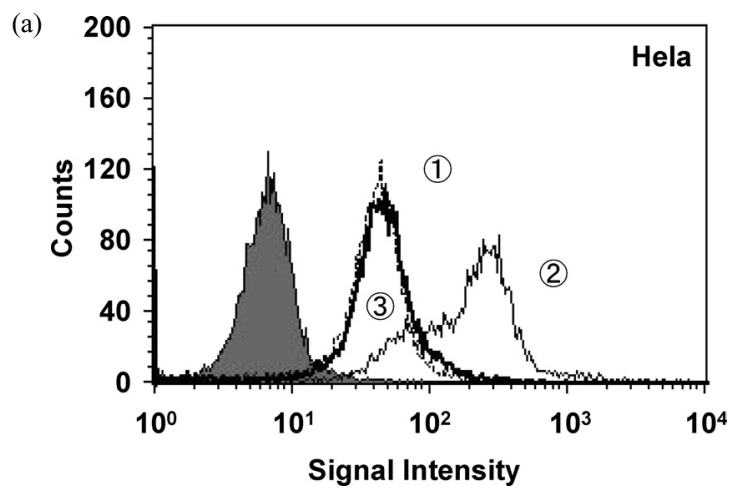

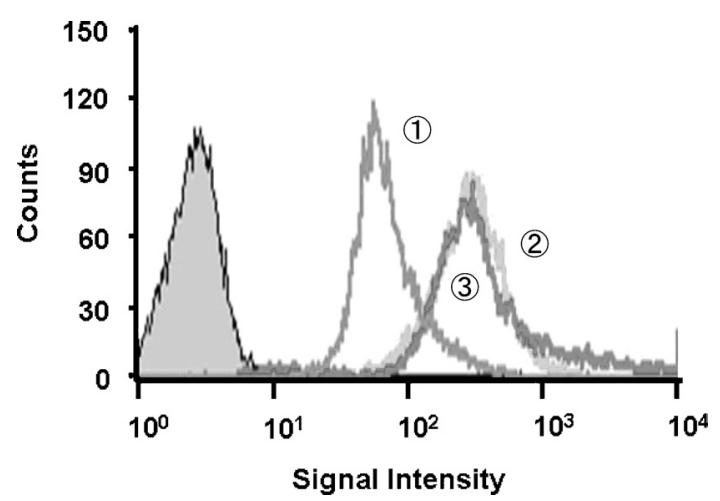

Fig. 5. Intracellular Penetrating Activity of FITC-Labeled CPPs on A431 Cells

Ten micromolar FITC-labeled 439A peptide (1), 435B peptide (2) and parent Tat peptide (3) were added to A431 cells. Gray area showed the distribution of non-treated cells. Following trypsinization, the quantity of penetrating peptide was evaluated in cells according the level of fluorescence intensity.

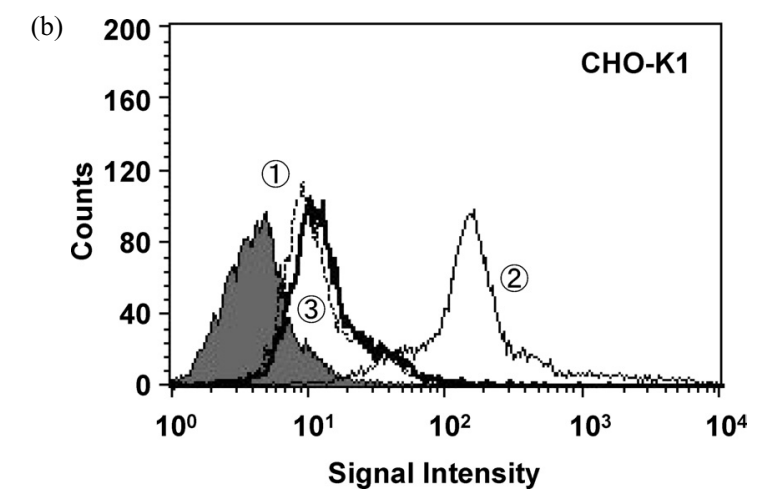

Fig. 6. Intracellular Penetrating Activity of FITC-Labeled CPPs on Human and Murine Cell Lines

Ten micromolar FITC-labeled 439A peptide (1), 435B peptide (2) and parent Tat peptide (3) were added to Hela (a) and CHO (b) cells. Gray area showed the distribution of non-treated cells. Following trypsinization, the quantity of penetrating peptide was evaluated in cells according the level of fluorescence intensity.

cells, the efficiency in terms of cell penetration between the parent Tat peptide and 435B peptide was almost the same, though the penetrating activity of the 439A peptide was decreased about 10 fold compared to the parent Tat peptide and 435B peptide (Fig. 5). However, on Hela and CHO cells, the efficiency of cell penetration between the parent Tat peptide and 439A peptide was almost the same, though the penetrating activity of the 435B peptide was increased about 10 fold compared to the parent Tat peptide and 439A peptide (Figs. $6 a, b)$.

Inhibition of Endocytic Internalization Several studies were done to investigate the involvement of macropinocytosis or caveolae/raft-dependent endocytosis on peptide transduction domain such as TAT. The effect of the specific macropinocytosis inhibitor, amiloride, on TAT peptide penetration was determined. As seen in Fig. 7, treatment with amiloride did not inhibit 435B and 439A pepetration. Additionally, Methyl- $\beta$-cyclodextrin ( $\mathrm{M} \beta \mathrm{CD}$ )-sensitive caveolae/ raft-dependent endocytosis of $435 \mathrm{~B}$ and 439A peptides was detected, internalization of $435 \mathrm{~B}$ and 439A via transduction is significantly affected by $\mathrm{M} \beta \mathrm{CD}$ treatment.

\section{DISCUSSION}

In an effort to search for novel CPPs, we have screened an

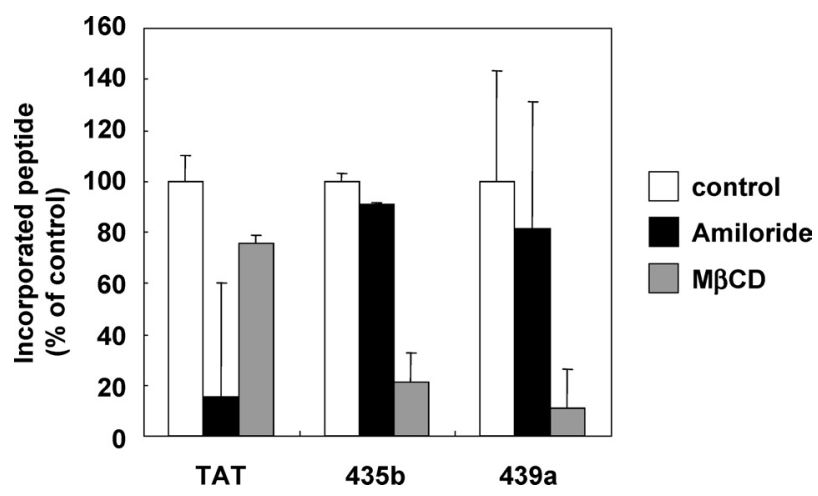

Fig. 7. Inhibitory Effects of Amiloride and $\mathrm{M} \beta \mathrm{CD}$ for Peptide Incorporation on A431 Cells

FITC-labeled 435B peptide and 439A peptide were added to cells after amiloride and $\mathrm{M} \beta \mathrm{CD}$ pre-incubated. The quantity of penetrating peptide was evaluated by FACS analysis according the mean of fluorescence intensity.

M13 peptide phage display library comprised of CPPs based on the Tat transduction domain. From this screening approach, we have identified peptides that displayed a capability for cell penetration. In particular, a screen using A431 cells resulted in identification of peptide 435B and 439A that is able to penetrate cultured cells in vitro efficiently. Thus, this method of screening for CPPs using phage peptide li- 
braries can be readily applied to select from a wide variety of possible peptides.

Whether the parent Tat peptide shows the penetrating capability of other known CPPs is uncertain. ${ }^{12,16)}$ The RGD peptide has long been used to facilitate the transport of bioactive molecules through adsorptive endocytosis. ${ }^{17)}$ However, comparison of short oligolysine peptides to that of equivalent length polymers of arginine showed that oligoarginine was much more efficient in carrying GFP into cultured cells. ${ }^{18)}$ Conversely, the ability of short oligolysine peptides, from 6 to 12 residues in length, has been shown to be more efficient than oligoarginine in carrying larger macromolecules $(60-500 \mathrm{kDa})$ into cultured cells. In this study, the 435B and 439A peptides contained hardly any basic amino acids but still displayed an ability to mediate cell penetration more efficiently than the parent Tat peptide. This result indicated that the cell penetrating activity of CPPs is controlled not only by the electrical charge but also by the structural characteristics. Practically, it is known that the amino acid component and associated tertiary structure of peptides are essential for cell penetrating activity. ${ }^{19)}$ We did not examine our peptides on a structural level; however, the results presented here suggest the importance of hydrophobicity, as hydrophobic amino acids were enriched by sequential biopanning.

It has been theorized that the ionic interaction between positively charged Arg residues in these CPPs and the negatively charged phosphate head group of the membrane lipid bilayer plays a key role in CPP membrane interaction. ${ }^{20)}$ However, the exact mechanism by which these CPPs operate is still largely unknown. Work performed by several investigators has shown that Tat binds heparin and that this heparin/Tat interaction involves the basic domain of Tat. ${ }^{21)}$ Meanwhile, previous study showed that the histidine residues of peptide sequence might enhance an endosomal escape of the cargo. ${ }^{22}$ In this study, the $435 \mathrm{~A}$ and $439 \mathrm{~B}$ peptides did not show enrichment of basic amino acids, but did exhibit an increase in proportion of hydrophobic amino acids. Several numbers of histidine residues were included in $435 \mathrm{~B}$ and 439A peptide sequence compared to native TAT peptide. Accordingly, it is possible that these peptides do not penetrate through the binding of cell surface receptors such as HSPG but escape from endosome efficiently. Whereas the conformation of these peptides should be examined, our results suggest that the $435 \mathrm{~B}$ and $439 \mathrm{~A}$ peptides penetrate the cell membrane independently of cell surface receptor.

In this study, the transduction efficiency was observed to be different between the peptides fused with PSIF and those labeled with FITC. We think there are two ideas to explain this discrepancy. Firstly, the molecular size and structure of the respective cargo is different. Secondly, there is some possibility that the intracellular kinetics is different between the parent Tat peptide and our peptides. It is thought that the parent Tat peptide is transferred to nuclei after penetrating the cell membrane, while our mutant peptide-PSIF conjugate diffuses throughout the cytoplasm. We are currently examining the intracellular kinetics of these peptides in an effort to resolve this issue.

In this study, our peptides have a unique sequence compared to preexisting CPPs. These peptides are able to intro- duce a large molecule into the intracellular space more efficiently than the parent Tat peptide, the latter which is known to have a high level of transduction ability. Using these peptides, efficient introduction of large molecules to the cytoplasm is accomplished. As such, one could readily conceive of using these peptides to target disease-related proteins, revealed from extensive-omic analysis. Furthermore, our peptides can be used as analytical tools to explore the mecha$\operatorname{nism}(\mathrm{s})$ of peptide penetration.

Acknowledgements This study was supported by the following grants: a Grant-in-Aid for Scientific Research (No. 17689008, 17016084, 17790135, 16790534, 18015055, 18659047) from the Ministry of Education, Culture, Sports, Science and Technology of Japan; a Grant-in-Aid for Scientific Research from the Japan Society for the Promotion of Science; Health and Labour Sciences Research Grants from the Ministry of Health, Labour; a Research Grant from the New Energy and Industrial Technology Development Organization (NEDO; No. 03A47016a), and JSPS Research Fellowships for Young Scientists (No. 08476, 08841, 09131) from the Japan Society for the Promotion of Science.

\section{REFERENCES}

1) Frankel A. D., Pabo C. O., Cell, 55, 1189-1193 (1988).

2) Derossi D., Joliot A. H., Chassaing G., Prochiantz A., J. Biol. Chem., 269, 10444-10450 (1994).

3) Futaki S., Suzuki T., Ohashi W., Yagami T., Tanaka S., Ueda K., Sugiura Y., J. Biol. Chem., 276, 5836-5840 (2001).

4) Lewin M., Carlesso N., Tung C. H., Tang X. W., Cory D., Scadden D. T., Weissleder R., Nat. Biotechnol., 18, 410-414 (2000).

5) Ryu J., Lee H. J., Kim K. A., Lee J. Y., Lee K. S., Park J., Choi S. Y., Mol. Cells, 17, 353-359 (2004).

6) Schwarze S. R., Ho A., Vocero-Akbani A., Dowdy S. F., Science, 285, 1569-1572 (1999).

7) Zorko M., Langel U., Adv. Drug Deliv. Rev., 57, 529-545 (2005).

8) Wadia J. S., Dowdy S. F., Adv. Drug Deliv. Rev., 57, 579-596 (2005).

9) Ferrari A., Pellegrini V., Arcangeli C., Fittipaldi A., Giacca M., Beltram F., Mol. Ther, 8, 284-294 (2003).

10) Wadia J. S., Stan R. V., Dowdy S. F., Nat. Med., 10, 310-315 (2004).

11) Richard J. P., Melikov K., Brooks H., Prevot P., Lebleu B., Chernomordik L. V., J. Biol. Chem., 280, 15300-15306 (2005).

12) Brooks H., Lebleu B., Vives E., Adv. Drug Deliv. Rev., 57, 559-577 (2005).

13) Lindgren M., Hallbrink M., Prochiantz A., Langel U., Trends Pharmacol. Sci., 21, 99-103 (2000).

14) Yamamoto Y., Tsutsumi Y., Yoshioka Y., Nishibata T., Kobayashi K., Okamoto T., Mukai Y., Shimizu T., Nakagawa S., Nagata S., Mayumi T., Nat. Biotechnol., 21, 546-552 (2003).

15) Shibata H., Yoshioka Y., Ikemizu S., Kobayashi K., Yamamoto Y., Mukai Y., Okamoto T., Taniai M., Kawamura M., Abe Y., Nakagawa S., Hayakawa T., Nagata S., Yamagata Y., Mayumi T., Kamada H., Tsutsumi Y., Clin. Cancer Res., 10, 8293-8300 (2004).

16) Futaki S., Int. J. Pharm., 245, 1-7 (2002).

17) Gresham H. D., Goodwin J. L., Allen P. M., Anderson D. C., Brown E. J., J. Cell Biol., 108, 1935-1943 (1989).

18) Han K., Jeon M. J., Kim S. H., Ki D., Bahn J. H., Lee K. S., Park J., Choi S. Y., Mol. Cells, 12, 267-271 (2001).

19) Lindberg M., Jarvet J., Langel U., Graslund A., Biochemistry, 40, $3141-3149$ (2001).

20) Ziegler A., Blatter X. L., Seelig A., Seelig J., Biochemistry, 42, 9185-9194 (2003).

21) Rusnati M., Tulipano G., Urbinati C., Tanghetti E., Giuliani R., Giacca M., Ciomei M., Corallini A., Presta M., J. Biol. Chem., 273, $16027-$ 16037 (1998).

22) Midoux P., Monsigny M., Bioconjug. Chem., 10, 406-411 (1999) 